\title{
Real-time operation of a centralized Energy Management System for an islanded Microgrid
}

\author{
A. Peña Asensio ${ }^{1}$, S. Arnaltes Gómez ${ }^{1}$, J.L. Rodriguez-Amenedo ${ }^{1}$, M. Garcia-Plaza ${ }^{1}$, M. Prodanovic ${ }^{2}$ \\ ${ }^{1}$ Department of Electrical Engineering \\ Carlos III University, Leganes, Madrid (Spain) \\ Phone number: +34 916248851, e-mail: anpenaa@ing.uc3m.es \\ ${ }^{2}$ Institute IMDEA Energy, Madrid, (Spain) \\ e-mail: milan.prodanovic@imdea.org
}

\begin{abstract}
With an increasing impact of microgrids (MGs) on modern power systems it is necessary to have algorithms ready and flexibly adaptable to different operating conditions and energy scenarios. This paper proposes an algorithm for the realtime operation of an islanded MG that is not based on demand and generation prediction. The objective of a centralized Energy Management System is to minimize the use of conventional, fuel based generation by using the State of Charge of the deployed Battery Energy Storage System to indicate the operating state of the MG.
\end{abstract}

\section{Key words.}

Micro-grids, Smart grids, Energy Management Systems, Distributed generation, Hybrid Systems.

\section{Introduction}

Microgrids (MGs) are changing the way we look at power systems. They usually comprise multiple load, distributed generation and energy storage (EESs) systems. MGs can work connected to a main distribution grid, joining all of its elements into a single unit, or disconnected in the socalled isolated operation [1].

Isolated operation of MGs can be of great importance for actual and future power systems. Applications vary from back-up systems on critical infrastructures to users that want to disconnect from the grid in order to manage their own energy consumption. They present a feasible way of merging different DGSs, thus improving renewable energy integration [2], [3]. This can be of special importance on remote systems, such as islands, due to the increased cost of fuel on these systems [4].

Optimization on the use of Renewable Energy Generation Systems (REGSs) through EESs, despite its uncertainty [5], is one of the most critical aspects [6]. A common way to address this issue is using a centralized controlling unit called the Energy Management System (EMS) [7].

EMSs have been widely studied in the literature as high level coordinating elements in MG applications. Most studies focus on the optimization of the MG operation through linear programming applied to different time horizons. [8]-[11].

These studies are based on the availability of information from forecast systems and from the MG elements. However, precision of forecast, both for load and renewable energy systems, is still a developing topic [12]. Moreover, despite the deployment of smart metering systems, real-time information from load demand is still unavailable in most systems [13].

In this paper, a different approach is proposed. Assuming that the MG has a Battery ESS (BESS), although similar approaches could be made for other ESSs, the EMS uses the BESS State of Charge (SOC) as a measurement of the MG state. Thus, no load or forecast information is needed.

Using this information, the EMS minimizes the use of Conventional Generation Systems (CGSs). Note that this is a realistic approximation to an island or remote system where, as stated earlier, cost of fuel is especially significant.

In Section 2, this algorithm is presented, highlighting its main parameters and characteristics. In Section 3 the implementation of the algorithm on the case of a specific microgrid is discussed. Finally, Section 4 presents the results obtained from this implementation.

The structure of the microgrid case under study is depicted Fig. 1. 


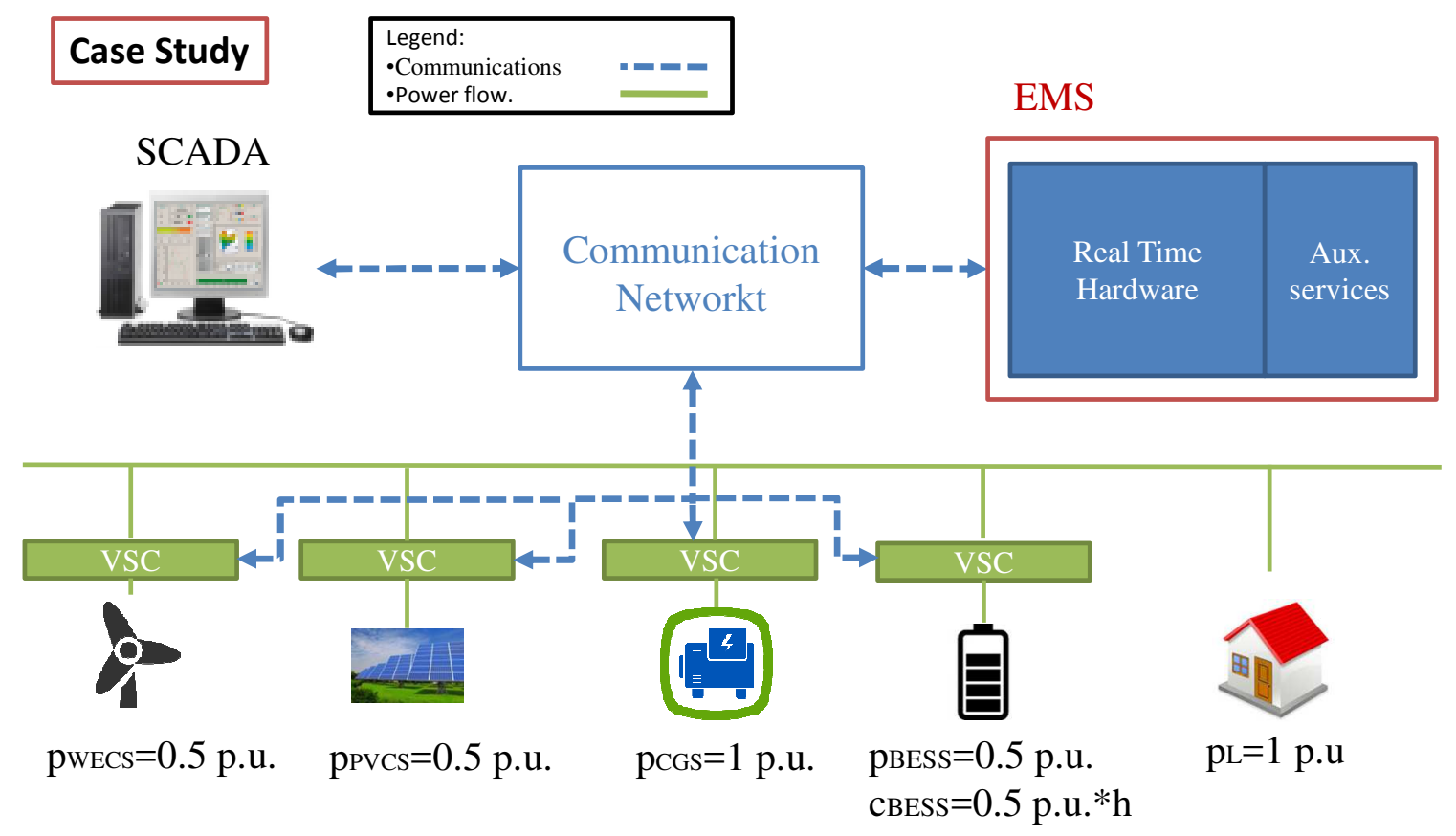

Fig. 1 System proposed in the paper.

\section{EMS Operation}

The proposed EMS algorithm is designed to minimize the use of CGSs in an isolated MG. As stated in the introduction, this could be interesting when fuel costs, including damage to the environment, are considered specially high, which is usually the case on islanded systems [4].

To achieve this objective, the EMS should prioritize the use of REGSs. Moreover, the EMS should be able to turn off the CGS when it is not needed to supply the loads.

A BESS is used to store the REGSs energy that is not demanded by the loads so it can be used when REGSs are not available. In this way, REGSs use is optimized. The optimization will depend on the BESS storage capacity. This application is usually referred to as load-shifting [14].

In order to turn off the CGSs the EMS must ensure the MG will remain stable without them, considering that CGSs usually have restrictions in turn-on times [15]. A common way to ensure this, within a certain confidence margin, is to use forecast models [12].

In this paper, a different approach is proposed where the BESS State Of Charge (SOC) is used as a measurement of the MG state. In this way, a high SOC indicates that REGSs are available while a low SOC indicates the need for CGSs. Moreover, a high SOC indicates the BESS is capable of supporting the grid even if REGSs power is low. It is, therefore, a reliable indicator the CGSs can be safely turned off.

\section{A. Proposed algorithm description}

The EMS can actuate in the CGSs, by forcing an active power set-point or by turning them off, and on the REGSs, by limiting its active power.
The set-point for the CGSs should be the difference between the REGSs power and the measured load power. However, as stated in the introduction, load power information is not usually available in real-time.

In this paper, it is assumed that load power demand is not available in real-time. Instead, the BESS SOC information will be used to determine the power requirements.

Assuming the BESS is controlled to balance the MG power, either by a grid forming or droop control scheme [16], [17], the SOC should rise when there is an excess of renewable energy and otherwise decrease to avoid charging the BESS by CGSs.

Following this principle, the set-points for CGSs generation (subscript $D$ ) and REGSs limitation (subscript $R$ ) are calculated through a PI control strategy as

$$
\begin{gathered}
p_{C}{ }^{*}=k_{p C}\left(\operatorname{SOC}_{C}{ }^{*}-S O C\right)+k_{i C} \cdot x_{C}, \\
p_{R}{ }^{*}=k_{p R}\left(\operatorname{SOC}_{R}{ }^{*}-S O C\right)+k_{i R} \cdot x_{R},
\end{gathered}
$$

where $k_{p}$ and $k_{i}$ are the controller proportional and integral gains, respectively and $x$ is the controller error state, given by

$$
\begin{aligned}
& \frac{1}{\omega_{0}} \frac{d}{d t} x_{C}=\operatorname{SOC}_{C}{ }^{*-S O C}, \\
& \frac{1}{\omega_{0}} \frac{d}{d t} x_{R}=\operatorname{SOC}_{R}{ }^{*-S O C} .
\end{aligned}
$$

SOC values are expressed as percentage of the rated BESS capacity $\left(C_{N}\right)$. All other values are written in per 
unit relative to the nominal $\mathrm{MG}$ power $\left(P_{N}\right)$, frequency $\left(\omega_{0}\right)$ and voltage $\left(V_{N}\right)$.

If the SOC is increased beyond $\mathrm{SOC}_{\mathrm{R}}{ }^{*}$ the EMS will actuate limiting the REGSs power. Likewise, if the SOC drops below $\mathrm{SOC}_{\mathrm{C}}{ }^{*}$, an active power set-point will be sent to the CGSs.

Given that $\mathrm{SOC}_{\mathrm{R}}{ }^{*}$, is greater than $\mathrm{SOC}_{\mathrm{C}}{ }^{*}$, the controller will try to charge the BESS to $\mathrm{SOC}_{\mathrm{R}}{ }^{*}$ by using REGSs. When it is no possible, it will try to keep the SOC at safe operating point $\mathrm{SOC}_{\mathrm{C}}{ }^{*}$.

Since EMS actuations are limited by the communication delays, the BESS internal control will assume the instantaneous load or REGSs power variations. The reference SOC for each controller should be selected considering that a certain SOC margin should be kept to compensate for these variations. These margins as well as the $S O C_{C}$ and $S O C_{R}$ relative values are depicted in Fig. 2Fig. 2 .

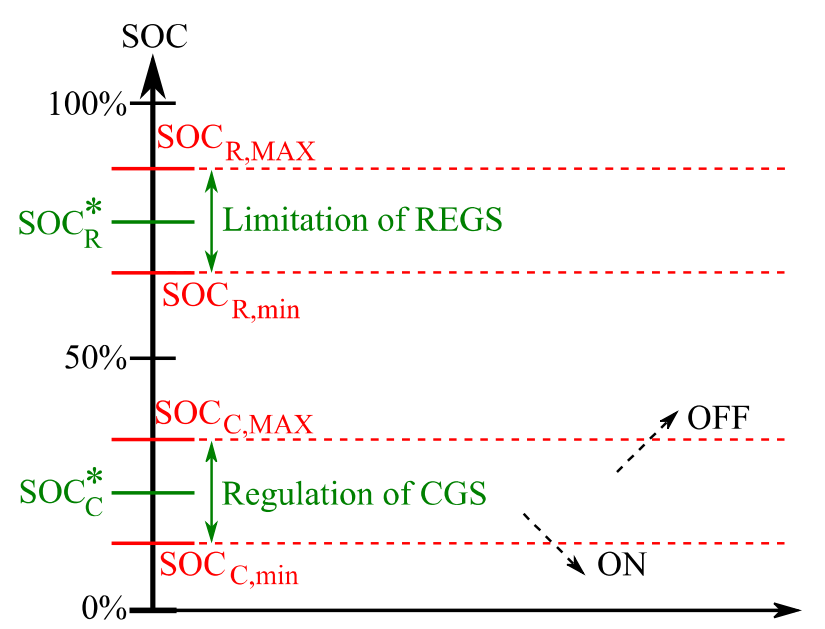

Fig. 2 Relative positions of the SOC controller references.

Each controller should be disabled when the SOC falls outside of its respective margins to avoid interaction between controllers as well as wind-up situations. This is automatically achieved through the saturation of the EMS controllers.

$S O C_{R, M A X}$ and $S O C_{R, \min }$ represents the margin at which the BESS should operate while limiting the RESs power.

The CGSs can be turned off when the SOC is increased beyond $S O C_{C, M A X}$ as it would mean that there is renewable energy available. For this purpouse $S O C_{C, M A X}$ should ensure that the BESS can support the load for, at least, the time that CGSs need to turn on. $S O C_{C \text {, min }}$ represents the limit at which the BESS should be discharged due to technical or economical reasons.

The selection of these set-points will determine the BESS use and thus further optimization of the system can be achieved by an on-line variation.

\section{Case study}

This section analyses the considerations required for the application of the proposed algorithm to a specific islanded system. A scheme of this system is depicted in Fig. 1Fig. 1.

The EMS is implemented on a real-time platform. Since it has to communicate with the different elements, the communication delays must be taken into account for the execution time selection and the PI tuning.

The MG includes a diesel-fueled CGS, a BESS and both wind and solar REGSs. A brief description of each system is given in order to highlight the characteristics of the proposed algorithm. Note that both the optimal sizing and the detailed modeling of each system are out of the scope of this paper.

\section{A. Load}

The load is modelled as a constant power demand $p_{L}$ with a maximum peak value of 1 p.u. There is no communication between the load and the EMS.

\section{B. $C G S$}

The CGS is modeled as a diesel-fed generating unit with a controllable power generation set-point $p_{C}$. It is considered that this system can support the load on its own and thus has a rated power of 1 p.u.

It is considered that it can follow the EMS active power reference, $p_{C}{ }^{*}$, in a time that does not affect the EMS controllers bandwidth and thus its variations can be considered as instantaneous from the EMS point of view.

Based on [15], a technical minimum, $p_{C, \min }$, of $20 \%$ of the rated power is considered. The turn-on time is modelled as a ramped variation of the active power from 0 to $p_{C, \min }$ over 10 minutes.

\section{REGSS}

Both the wind and solar REGSs are sized to 0.5 p.u.. This is in order to allow situations when the load is entirely supplied by REGSs. A photovoltaic conversion system (PVCS) and a wind energy conversion system (WECS) are combined to obtain a more constant power profile.

The EMS is responsible for sharing the power reference $p_{R}{ }^{*}$ between the solar and wind generation systems. No economic discrimination is considered and thus the allocation depends only on the REGSs availability. The combination of both sources is then integrated into the REGSs power $p_{R}$.

It is considered REGSs can follow the EMS set-point instantaneously, in the same way as CGSs.

\section{BESS}

The BESS power, $p_{B E S S}$, is sized to 0.5 p.u. This is an approximation to cover scenarios of load or REGSs 
power variations that are faster than the EMS actuation times.

The BESS energy is sized considering the worst-case scenario in which the CGS is disconnected (SOC at $\left.S O C_{D, M A X}\right)$ and there is not enough renewable energy to cover the load demand for the time that the CGS takes to reconnect. In fact, this is not a situation that is likely to happen and thus a more optimal BESS energy sizing could be achieved considering historical data on the installation.

The BESS SOC is modelled by using an ampere-counting solution [18]. Although there are more accurate techniques, such as extended Kalman filters [19], amperecounting algorithms are very extended due to their simplicity. Considering that, in the designed SOC margin, the BESS voltage does not vary, power can be used instead of current in the ampere-counting model.

As stated in Section 2, the internal control of the BESS is designed to act as a slack node for the MG. Thus, any mismatch between generation and load is immediately compensated by using energy from the BESS.

\section{Result and discussion}

The system presented in Section 3 was tested on simulation using MATLAB ${ }^{\circledR}$ Simulink $^{\circledR}$.

To analyse the EMS operation in a realistic situation, variations of wind, PV and load power were considered over a period of 2 days, allowing multiple energy scenarios to be included. The considered profiles are depicted in Fig. 3Fig. 3 .
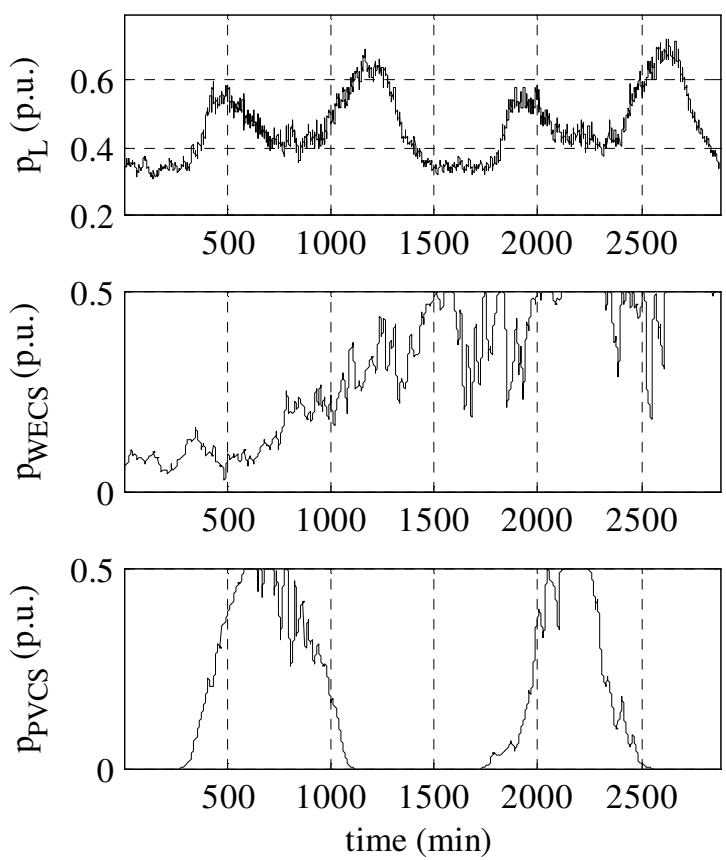

Fig. 3 Load and REGs power profiles.

As stated in Section 3, the EMS execution period, $\mathrm{T}_{\mathrm{EMS}}$, should be selected considering the expected communication delays. Considering delays of up to
1 second, representing a good approximation in $\mathrm{MG}$ scenarios [20], the execution time was fixed at 4 seconds.

Since the EMS must be operated in real-time, to avoid long simulations, the 2-days profiles of Fig. 3 Fig. 3 were executed in 2 minutes.

The BESS energy and diesel turn-on times were sized accordingly with this time transformation. Note that the EMS algorithm will be exposed to more demanding power variations than in the 2-day scenario. The main parameters used for the simulation are summarized in Table IFable .

Table I - Simulation parameters

\begin{tabular}{|l|l|l|l|}
\hline Label & Value & Unit & \multicolumn{1}{c|}{ Description } \\
\hline $\mathrm{P}_{\mathrm{N}}$ & 25 & $\mathrm{~kW}$ & MG nominal power. \\
\hline $\mathrm{T}_{\mathrm{S}}$ & 50 & $\mathrm{~ms}$ & Simulation step period. \\
\hline $\mathrm{T}_{\mathrm{EMS}}$ & 4 & $\mathrm{~s}$ & EMS execution period. \\
\hline$\alpha_{\mathrm{t}}$ & 60 & - & $\begin{array}{l}\text { Relation between simulated and } \\
\text { real time. }\end{array}$ \\
\hline $\mathrm{k}_{\mathrm{pC}}$ & 0.04 & - & CGS controller proportional gain. \\
\hline $\mathrm{T}_{\mathrm{C}}$ & 40 & $\mathrm{~s}$ & CGS controller time constant. \\
\hline $\mathrm{k}_{\mathrm{pR}}$ & 0.04 & - & $\begin{array}{l}\text { REGS controller proportional } \\
\text { gain. }\end{array}$ \\
\hline $\mathrm{T}_{\mathrm{R}}$ & 40 & $\mathrm{~s}$ & REGS controller time constant. \\
\hline
\end{tabular}

The system response to the profiles of Fig. 3Fig. 3 is depicted in Fig. 4Fig. 4. The BESS power is considered positive for charging and negative for discharging. To address the operation of the proposed algorithm, two situations ( $\mathbf{A}$ and $\mathbf{B}$ ) have been highlighted.

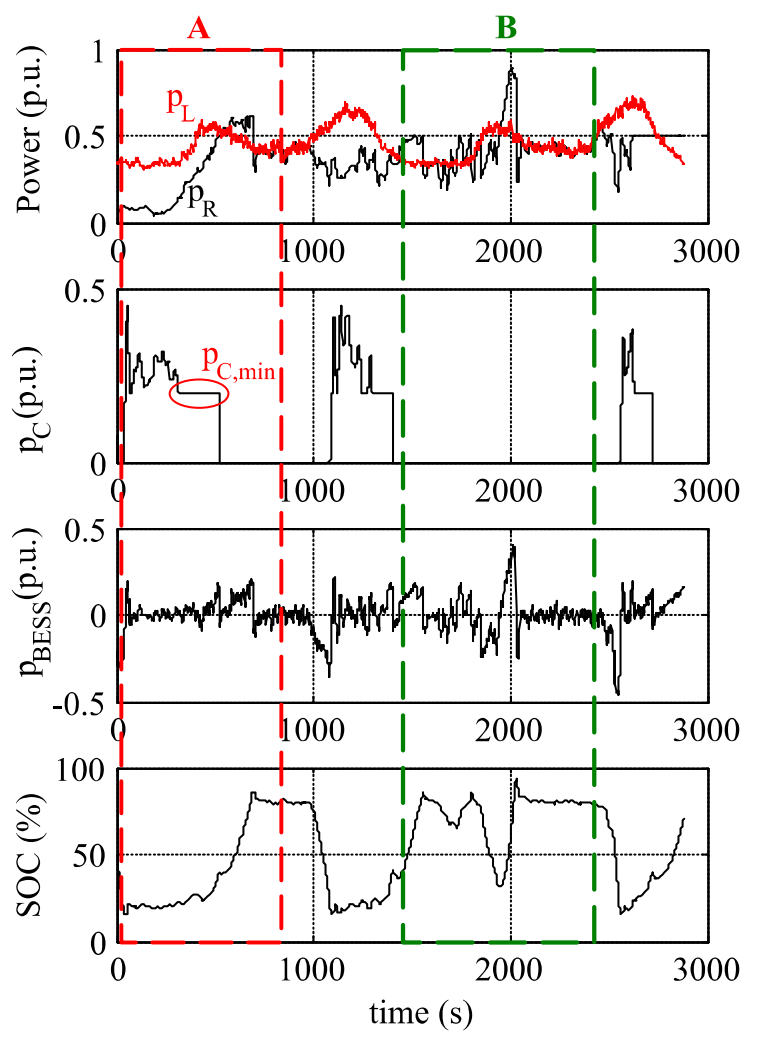

Fig. 4 Results during 2 days of operation. 
In $\mathbf{A}$, the simulation starts during a night with low REGSs power and thus the CGS must be activated. Due to the SOC regulation, the CGS power is not used to charge the battery but only to track the load demand.

When the load decreases and the solar energy becomes available, the SOC begins to increase. When there is enough energy stored, the CGS is turned off $\left(p_{C}=0\right)$ and the SOC regulation to $\mathrm{SOC}_{\mathrm{R}}{ }^{*}$ begins to actuate. Note that the CGS cannot be reduced below $p_{C \text {, min }}$ unless it is turned off. These transitions are performed automatically through the EMS controllers saturation.

A zoom of the regulation process in $\mathbf{A}$ is depicted in Fig. 5Fig. 5. When the SOC enters the actuation zone, the EMS starts to limit the REGSs power in order to track $\mathrm{SOC}_{\mathrm{R}}{ }^{*}$. When $p_{R}$ decreases, the BESS is discharged and the regulation is deactivated.

It can be seen how the load varies faster than the EMS actuation, represented by $p_{R}$ steps. As stated in Section 2, these variations are compensated through the BESS internal regulation, producing SOC variations around the reference value.
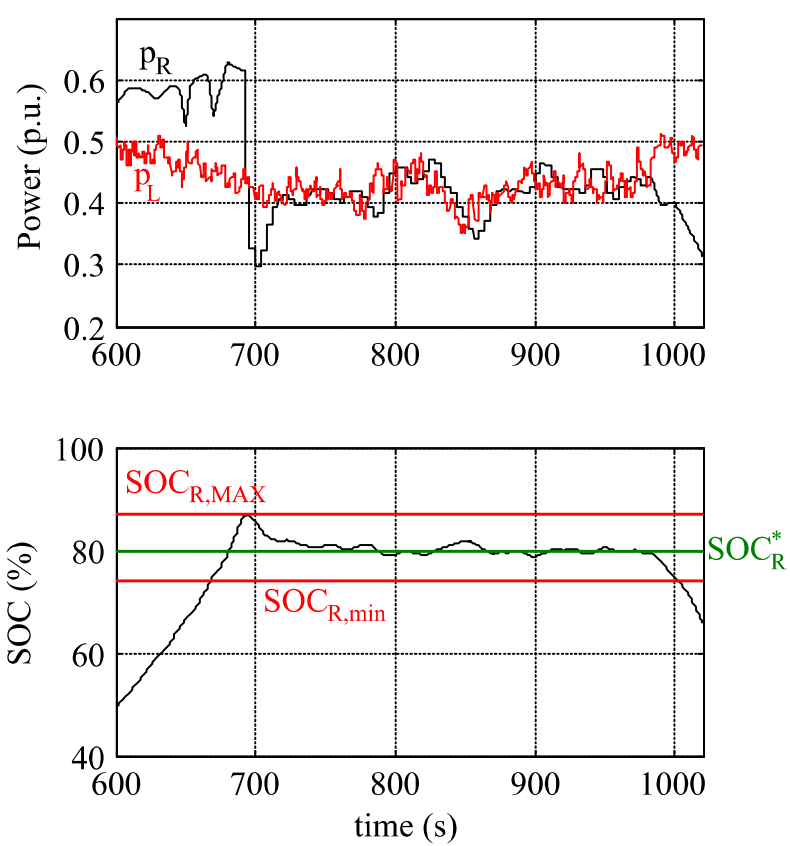

Fig. 5 Zoom of variables during period $\mathbf{A}$.

In $\mathbf{B}$, due to the high wind speeds, there is an excess of energy and the BESS is charged during the night (Fig. 6Fig. 6). This allows the EMS to avoid using the CGS during the first hours of demand, as happened in $\mathbf{A}$, so the load can be supplied entirely by the REGSs.

Despite the lack of CGSs, the SOC regulation allows the MG to follow the load variations thus ensuring a stable operation.

In Fig. 6Fig. 6 it can be seen how the algorithm performs the transitions in and out of REGSs limitation seamlessly.
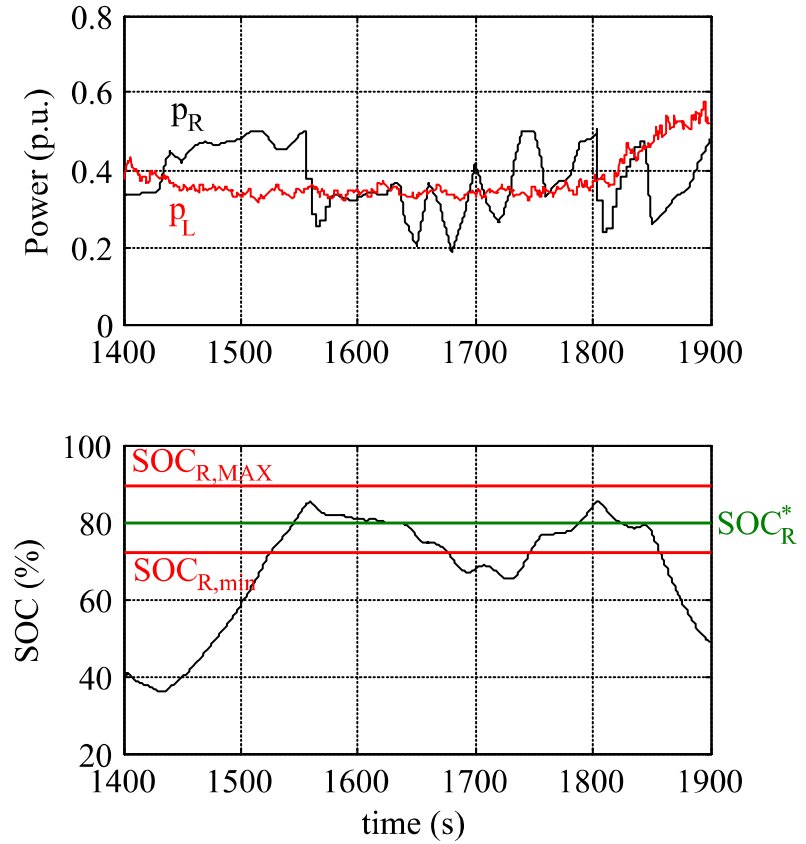

Fig. 6 Zoom of variables during period $\mathbf{B}$.

\section{Conclusion}

An algorithm for the real-time operation of an islanded MG without forecast or load demand information has been presented. Assuming the BESS is designed to balance instantaneous mismatches between generation and demand, it is shown how the BESS SOC can be used as a measurement of the MG state.

Using this information, the proposed algorithm decides the set-points for the CGSs and for the REGSs limitation in order to follow load variations. CGSs are activated only when the SOC is low while REGSs are only limited when it is high. Transitions between these operation modes are performed automatically by the saturation of the algorithm controllers.

Simulations results show how this strategy ensures the stable operation of the MG under multiple load and renewable energy scenarios.

The algorithm is designed to minimize the use CGSs. Moreover, simulation results show that the EMS is able to turn off CGSs without compromising the MG operation. Thus, this algorithm is specially indicated for situations where fuel costs, including damage caused to the environment, are considered to be especially high, that is a common case in islanded systems.

\section{Acknowledgement}

This work has been supported by the Autonomous Community of Madrid under the PRICAM project (S2013-ICE-2933). 


\section{References}

[1] R. H. Lasseter, 'MicroGrids', in IEEE Power Engineering Society Winter Meeting, 2002, 2002, vol. 1, pp. 305-308.

[2] M. S. Mahmoud and F. M. Al-Sunni, Control and Optimization of Distributed Generation Systems. Springer, 2015.

[3] P. Piagi and R. H. Lasseter, 'Autonomous control of microgrids', in 2006 IEEE Power Engineering Society General Meeting, 2006, p. 8 pp.-.

[4] D. Weisser, 'On the economics of electricity consumption in small island developing states: a role for renewable energy technologies?', Energy Policy, vol. 32, no. 1, pp. 127-140, Jan. 2004.

[5] W.-G. Früh, 'From local wind energy resource to national wind power production', AIMS Energy, vol. 3, no. 1, pp. 101-120, 2015.

[6] M. Muselli, G. Notton, and A. Louche, 'Design of hybridphotovoltaic power generator, with optimization of energy management', Sol. Energy, vol. 65, no. 3, pp. 143-157, 1999.

[7] H. Bevrani and T. Ise, Microgrid Dynamics and Control. John Wiley \& Sons, 2017.

[8] R. Firestone and C. Marnay, 'Energy manager design for microgrids', Lawrence Berkeley Natl. Lab., 2005.

[9] H. Kanchev, D. Lu, F. Colas, V. Lazarov, and B. Francois, 'Energy management and operational planning of a microgrid with a PV-based active generator for smart grid applications', IEEE Trans. Ind. Electron., vol. 58, no. 10, pp. 4583-4592, 2011.

[10] W. G. Früh, 'Optimising the use of a battery in a winddiesel-battery hybrid island grid using power prediction and day-ahead optimisation'.

[11] F. Katiraei, M. R. Iravani, and P. W. Lehn, 'Micro-grid autonomous operation during and subsequent to islanding process', IEEE Trans. Power Deliv., vol. 20, no. 1, pp. 248-257, Enero 2005.

[12] T. Hong and S. Fan, 'Probabilistic electric load forecasting: A tutorial review', Int. J. Forecast., vol. 32, no. 3, pp. 914-938, 2016.

[13] Y. Kabalci, 'A survey on smart metering and smart grid communication', Renew. Sustain. Energy Rev., vol. 57, no. Supplement C, pp. 302-318, May 2016.

[14] T. Kousksou, P. Bruel, A. Jamil, T. El Rhafiki, and Y. Zeraouli, 'Energy storage: Applications and challenges', Sol. Energy Mater. Sol. Cells, vol. 120, pp. 59-80, 2014.

[15] S. A. Papathanassiou and N. G. Boulaxis, 'Power limitations and energy yield evaluation for wind farms operating in island systems', Renew. Energy, vol. 31, no. 4, pp. 457-479, Apr. 2006.

[16] K. D. Brabandere, B. Bolsens, J. V. den Keybus, A. Woyte, J. Driesen, and R. Belmans, 'A Voltage and Frequency Droop Control Method for Parallel Inverters', IEEE Trans. Power Electron., vol. 22, no. 4, pp. 11071115, Jul. 2007.

[17] F. Katiraei, R. Iravani, N. Hatziargyriou, and A. Dimeas, 'Microgrids management', IEEE Power Energy Mag., vol. 6, no. 3, 2008.

[18] S. Piller, M. Perrin, and A. Jossen, 'Methods for state-ofcharge determination and their applications', J. Power Sources, vol. 96, no. 1, pp. 113-120, 2001.

[19] G. L. Plett, 'Extended Kalman filtering for battery management systems of LiPB-based HEV battery packs: Part 3. State and parameter estimation', J. Power Sources, vol. 134, no. 2, pp. 277-292, 2004.

[20] L. K. Siow, P. L. So, H. B. Gooi, F. L. Luo, C. J. Gajanayake, and Q. N. Vo, 'Wi-Fi based server in microgrid energy management system', in TENCON 2009-2009 IEEE Region 10 Conference, 2009, pp. 1-5. 